\title{
Artigo/Article
}

\section{Avaliação nutricional e alimentar de pacientes portadores de hanseníase tratados em unidades de saúde da grande Vitória, Estado do Espírito Santo}

\author{
The nutritional and dieting profiles of patients diagnosed with leprosy treated in the primary \\ healthcare units of Greater Vitória, State of Espírito Santo, Brazil
}

\author{
Rosa Maria Natalli Montenegro ${ }^{1}$, Maria Del Carmen Molina ${ }^{1}$, Marilda Moreira ${ }^{2}$ e Eliana Zandonade ${ }^{1}$
}

\begin{abstract}
RESUMO
Introdução: $\mathrm{O}$ presente estudo investigou a hanseníase quanto à apresentação clínica, perfil sociodemográfico, nutricional e alimentar de pacientes diagnosticados nas Unidades de Saúde dos municípios da Grande Vitória, Espírito Santo, Brasil, no período de janeiro a dezembro de 2009. Métodos: Foram estudados 152 pacientes de ambos os sexos, em início de tratamento poliquimioterápico. Foram coletados dados sociodemográficos, antropométricos, bioquímicos e de alimentação a partir de um questionário de frequência alimentar (QFA) validado e adaptado. Foi calculado o índice de massa corporal (IMC) para avaliação do estado nutricional. Resultados: O estudo mostrou que: 79 (52\%) dos participantes eram do sexo feminino, a média de idade foi de 40,4 anos $( \pm 16,9)$; $81(53,3 \%)$ possuíam vínculo empregatício; a média de anos de estudo foi de 7,1 $( \pm 4,5)$. Em relação à doença, 79 (52\%) eram multibacilares e $73(48 \%)$ paucibacilares. O índice baciloscópico foi negativo em $125(82,2 \%)$ pacientes. O excesso de peso foi identifcado em $11,8 \%$ e 5,3\% apresentaram baixo peso. $\mathrm{O}$ arroz e o feijão foram os alimentos relatados com maior frequência de consumo, $87,3 \%$ e $88,7 \%$ respectivamente. Conclusões: $\mathrm{O}$ estudo demonstrou que ações simples, iniciadas na atenção primária à saúde, podem auxiliar na melhoria do acompanhamento a portadores de hanseníase.
\end{abstract}

Palavras-chaves: Hanseníase. Avaliação nutricional. Avaliação alimentar

\begin{abstract}
Introduction: The present study investigated leprosy in relation to the clinical, sociodemographic, nutritional and dietary profiles of patients diagnosed at primary healthcare units in the suburbs of Greater Vitória, State of Espírito Santo, Brazil, from January to December 2009. Methods: The study involved 152 male and female patients initiating polychemotherapy treatment. The collected data included sociodemographic, anthropometric, biochemical and dietary aspects derived from a questionnaire of eating frequency (QEF) that was validated and adapted for Brazilian culture. Body mass index (BMI) was calculated to evaluate patient nutritional status. Results: The study showed that: 79 (52\%) of the participants were women, average age was 40.4 years-old $( \pm 16.9)$; $81(53.3 \%)$ were employed; the mean number of years of schooling was $7.1( \pm 4.5)$. Concerning the disease, $79(52 \%)$ of the participants were multibacillary and 73 (48\%) paucibacillary. The bacilloscopy index was negative in $125(82.2 \%)$ patients. Excess weight was identified in $11.8 \%$ and 5.3\% were underweight. Rice and beans were mentioned frequently, $87.3 \%$ and $88.7 \%$, respectively. Conclusions: The study showed that simple actions, initiated during primary healthcare, can help to improve the treatment of patients with the leprosy.
\end{abstract}

Keywords: Leprosy. Nutritional evaluation. Dieting profiles.

1. Programa de Pós Graduação em Saúde Coletiva, Universidade Federal do Espírito Santo, Vitória, ES 2. Programa de Hanseníase, Secretaria de Saúde do Estado do Espírito Santo, Vitória, ES.

Endereço para correspondência: Dra. Rosa Maria Natalli Montenegro. Rua Doutor Dido Fontes 40/201, Ed. Solar Menegatti, Jardim da Penha, 29060-280 Vitória, ES.

Tel: 5527 8838-2221

e-mail: rnnegro@hotmail.com

Recebido para publicação em 14/05/2010

Aceito em 04/11/2010

\section{INTRODUÇÃO}

A hanseníase, também conhecida como lepra, é uma doença infecciosa de evolução lenta e, no Brasil, permanece como um problema de saúde pública devido a sua magnitude, ao seu potencial incapacitante e por acometer a população na faixa etária ativa. O Espírito Santo é um estado endêmico para a hanseníase e esforços para o controle da doença têm sido realizados, com resultados positivos como a redução do coeficiente de prevalência de 32,6 casos em 1991 para 3,20 casos/10.000 habitantes em $2007^{1}$.

Atualmente, muitas discussões têm sido retomadas sobre a importância da nutrição nos cuidados com a saúde. Assim, a alimentação ganha importância na prevenção e controle de doenças. No contexto da saúde coletiva, estas devem estar inseridas nas ações dos direitos humanos para a vida e asseguradas pelas políticas públicas de promoção da saúde ${ }^{2}$.

O objetivo deste estudo foi caracterizar o perfil dos pacientes portadores da hanseníase quanto aos aspectos sociodemográficos, nutricionais e a forma de apresentação da doença em pacientes cadastrados nas Unidades de Saúde da Grande Vitória.

\section{MÉTODOS}

Trata-se de um estudo descritivo transversal de portadores de hanseníase em início de tratamento. Para o cálculo da amostra, utilizou-se os dados de população de pacientes com hanseníase nos municípios da Grande Vitória (Vitória, Vila Velha, Serra e Cariacica), no ano de 2007, num total de 400 casos novos $^{1}$ com proporção de eutróficos de aproximadamente $50 \%$. Considerando-se a precisão desejada de 6,5\% e o nível de significância de $5 \%$, o tamanho mínimo de amostra foi de 145 pacientes.

Os dados foram coletados nas unidades de saúde destes municípios por serem unidades com maior número de pacientes em registro ativo e 
todas estão localizadas na região metropolitana da grande Vitória, Espírito Santo.

No período de janeiro a dezembro de 2009, foram examinados todos os pacientes virgens de tratamento para a hanseníase ou os que tivessem iniciado a poliquimioterapia há um mês. Foram excluídos os pacientes coinfectados por tuberculose, aqueles que conhecessem serem portadores do vírus da síndrome de imunodeficiência adquirida e os pacientes com reações da hanseníase, pois as mesmas podem desencadear alterações sistêmicas, prejudicando a avaliação nutricional do paciente.

Os dados sociodemográficos foram obtidos por entrevista com auxílio de um formulário e os dados sobre a doença no prontuário clínico do paciente e transcritos para o instrumento de coleta. Os exames hematológicos (hematócrito e hemoglobina) foram solicitados como rotina do serviço no momento do diagnóstico e para análise destes indicadores foram utilizados os valores de referência preconizados por Porto ${ }^{3}$.

Para a avaliação antropométrica, foram coletados os seguintes dados: peso $(\mathrm{P})$, estatura $(\mathrm{E})$, circunferência do braço $(\mathrm{CB})$, dobra cutânea triciptal (DCT) e calculada a circunferência muscular do braço (CMB), interpretada conforme Frisancho.

Para a medida de DCT e CB, foi utilizado um adipômetro adequadamente calibrado, modelo Lange, e fita milimetrada, respectivamente, e obedecendo a critérios universalmente estabelecidos. Estas medidas foram analisadas isoladamente aplicando-se a tabela de percentis e interpretada conforme Frisancho ${ }^{4}$.

O índice de massa corpórea (IMC) foi calculado para a classificação do estado nutricional. Foi utilizada a classificação da Organização Mundial de Saúde ${ }^{5}$, para avaliar o estado nutricional dos adolescentes, adultos e idosos, por sexo.

Para avaliação da alimentação, foi utilizado o questionário de frequência de consumo alimentar (QFA) composto de 24 itens, validado por Kroke cols ${ }^{6}$ adaptado por Duarte ${ }^{7}$. Os alimentos foram classificados conforme as diretrizes do Guia Alimentar para a População Brasileira ${ }^{8}$.

A apresentação descritiva dos dados foi feita mediante análise estatística pelo programa SPSS, versão 17.0.

\section{Considerações éticas}

Esta pesquisa foi submetida ao Comitê de Ética da Universidade Federal do Espírito Santo, obtendo parecer favorável (processo 013/09). Foram atendidos os pressupostos éticos de pesquisa em seres humanos e mantido o sigilo das informações coletadas. Os pacientes assinaram o termo de consentimento livre e esclarecido.

\section{RESULTADOS}

Dos 152 pacientes participantes do estudo, $52 \%$ eram do sexo feminino. A idade variou de 10 a 74 anos, com média de 40,4 anos $( \pm 16,9)$. Do total de sujeitos, $53,3 \%$ possuíam vínculo empregatício e, em relação à renda salarial familiar, $11,2 \%$ das famílias recebiam menos de um salário mínimo; $64,3 \%$ de um a três salários mínimos; $17,8 \%$ de três até cinco salários mínimos e 6,6\%, mais de cinco salários mínimos.

Quanto à distribuição, segundo o município de residência, 14,5\% residiam em Cariacica, 23\% em Serra, 34,9\% em Vila Velha, 26,3\% em Vitória e 1,3\% em outros municípios. Em relação ao número de cômodos por domicílio, constatou-se que $21,1 \%$ dos pacientes possuíam de um a três cômodos na residência, $40,8 \%$ de três a cinco cômodos e $36,8 \%$ mais de cinco cômodos e um morador de rua $0,7 \%$. Em relação à quantidade de pessoas por domicílio, a média foi de $3,7( \pm 1,8)$ pessoas.

Quando avaliada a escolaridade, encontrou-se $11,4 \%$ analfabetos e a média de anos de estudo foi de 7,1 $( \pm 4,5)$.

Dos avaliados, quanto à classificação operacional (CO) preconizada pelo Ministério da Saúde, para fins de tratamento, $52 \%$ eram multibacilares (MB) e $48 \%$ paucibacilares (PB). Para a forma clínica (FC), segundo a classificação de Madri, encontrou-se: 9,2\% da forma clínica indeterminada (MHI), 42,8\% tuberculóide (MHT), $34,2 \%$ dimorfo (MHD) e 13,8\% virchowianos (MHV). O índice baciloscópico foi negativo em $82,2 \%$ dos pacientes.

A avaliação nutricional utilizando o método do IMC (índice de Quetelet) demonstrou que entre os estudados, 48,7\% foram classificados como eutróficos e 5,3\% como desnutridos. No grupo de sobrepeso, o percentual foi de $34,2 \%$ e no grupo de obesidade $11,8 \%$. A Figura 1 apresenta os resultados nas quatro categorias estudadas (baixo peso, eutrofismo, sobrepeso, obesidade) por sexo. Não se encontrou associação estatisticamente significante entre o sexo e IMC $(\mathrm{p}=0,250)$.

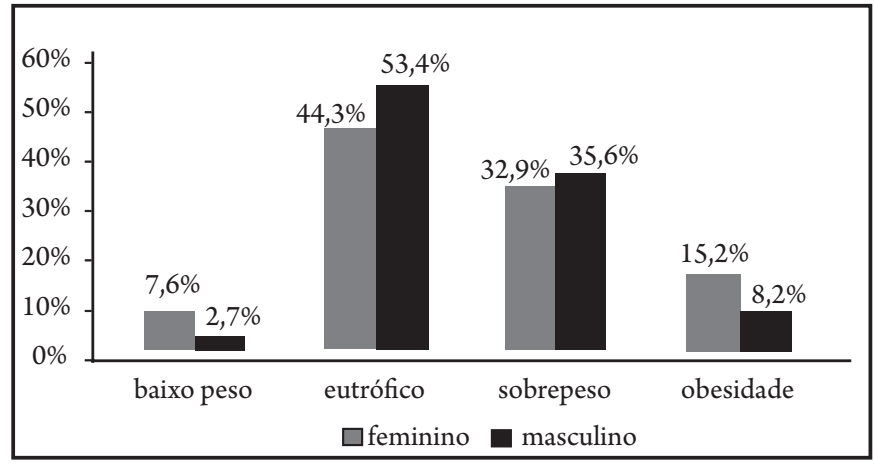

FIGURA 1 - Classificação segundo índice de massa corpórea e sexo.

A Tabela 1 apresenta os valores absolutos e percentuais das medidas de CB, DCT e CMB nas suas classificações (frequências absolutas e percentuais das classificações das variáveis $\mathrm{CB}, \mathrm{DCT}$ e $\mathrm{CMB}$ )

A classificação nutricional utilizando a $\mathrm{CB}$ avaliou o somatório dos componentes ósseo, muscular e de gordura e detectou um percentual de desnutrição igual a 30,3\%. A obesidade foi representada por $15,1 \%$ dos pacientes.

TABELA 1 - Frequências absolutas e percentuais das classificações das variáveis circunferência do braço, dobra cutânea tricipital e circunferência muscular do braço.

\begin{tabular}{|c|c|c|c|c|c|c|}
\hline \multirow[b]{2}{*}{ Classificação } & \multicolumn{2}{|c|}{$\mathrm{CB}$} & \multicolumn{2}{|c|}{ DCT } & \multicolumn{2}{|c|}{$\mathrm{CMB}$} \\
\hline & $\mathrm{n}^{\circ}$ & $\%$ & $\mathrm{n}^{\mathrm{o}}$ & $\%$ & $\mathrm{n}^{\mathrm{o}}$ & $\%$ \\
\hline Desnutrido grave & 1 & 7,0 & 35 & 23,0 & 3 & 2,0 \\
\hline Desnutrido moderado & 10 & 6,2 & 12 & 7,9 & 11 & 7,2 \\
\hline Desnutrido leve & 26 & 17,1 & 10 & 6,6 & 34 & 22,4 \\
\hline Eutrofia & 92 & 60,5 & 37 & 24,3 & 104 & 68,4 \\
\hline Sobrepeso & 9 & 5,9 & 15 & 9,9 & & * \\
\hline Obesidade & 14 & 9,2 & 43 & 28,3 & & * \\
\hline Total & 152 & 100,0 & 152 & 100,0 & 152 & 100,0 \\
\hline
\end{tabular}

CB: circunferência do braço, DCT: dobra cutânea tricipital, CMB: circunferência muscular do braço, * estas categorias não existem na classificação CMB. 
A classificação nutricional utilizando a medida da DCT demonstrou que $38,2 \%$ dos estudados apresentaram excesso de gordura, enquanto pelo cálculo da CMB, 31,6\% apresentaram baixo teor de massa muscular.

Em relação aos exames de hemoglobina, 62,5\% encontravamse na faixa de normalidade e 4,6 dos estudados apresentaram valor gravemente reduzido. Quanto ao hematócrito, os resultados são $69,7 \%$ e $3,9 \%$, respectivamente.

Foi aplicado o questionário de frequência alimentar para a amostra estudada. Dentre o grupo de cereais, tubérculos e raízes, observou-se que o arroz foi o alimento mais consumido, aparecendo no consumo diário de $87,3 \%$ da amostra. O consumo de frutas representou apenas $41,1 \%$ e o de vegetais $57 \%$. O feijão presente na dieta de $88,1 \%$ dos estudados com consumo de uma porção diária.

No grupo das proteínas, o alimento mais representativo foi o ovo (21,9\% do consumo diário). O leite estava presente no consumo semanal de 40,4\% dos estudados. Em relação à ingestão de carnes, observou-se um maior consumo semanal para o frango, com $88,7 \%$, e em segundo lugar a carne bovina, com $74,8 \%$.

As gorduras e os açúcares foram representados pelo consumo de frituras, manteiga e margarina, com $26,5 \%, 30,5 \%$ e $35,8 \%$, respectivamente, da amostra. Os açúcares foram representados pelo consumo diário de $31,8 \%$ dos estudados. Este grupo pode ser responsabilizado pelo excesso de gordura, em $38,2 \%$ dos examinados, na medida da dobra cutânea triciptal.

\section{DISCUSSÃO}

Do total de entrevistados, nos municípios da grande Vitória, ES, o percentual de acometimento pela doença foi ligeiramente maior no sexo feminino, com $52 \%$ dos casos, concordando com achados de estudo realizado em Bauru/SP9 9 . Quanto à faixa etária, observou-se um maior número de diagnóstico, na faixa etária de 40 a 49 anos. Outros estudos mostram a incidência predominante da doença, nessa faixa etária, como é o caso de estudo de perfil clínico realizado no nordeste do Brasil ${ }^{10}$. Na faixa etária de 10 a 19 anos, encontrou-se 25 pacientes diagnosticados, o que representando o percentual de $16,4 \%$ do total de casos.

A baixa escolaridade dos pacientes analisados, que dispunham de uma média de 7,1 $( \pm 4,5)$ anos de estudos, pode refletir-se em suas possibilidades profissionais de vida e emprego tendo como consequência, para a grande maioria, os baixos salários. $\mathrm{O}$ fenômeno da pauperização de pacientes, acometidos por doenças infecciosas, como é o caso da hanseníase, é discutido por diversos autores $^{11-12}$, que constatam que a infecção pelo Mycobacterium leprae acomete prioritariamente indivíduos que vivem em condições socioeconômicas desfavoráveis, como fatores que predispõem ao desenvolvimento da doença.

Poucos estudos avaliam estado nutricional em pessoas portadoras de hanseníase. Em nosso estudo, 74 pessoas, que representam $48,7 \%$ da amostra, foram classificadas como eutróficas, em relação à avaliação por IMC, resultado semelhante ao encontrado por Garcia' ${ }^{9}$, enquanto a avaliação do estado nutricional de pacientes portadores da doença em hospital na Argentina mostra um percentual de 23,7\% de eutróficos ${ }^{13}$.

Estudos do perfil nutricional da população brasileira mostram diferença nos valores de IMC quando relacionados com a idade e o sexo ${ }^{14}$, diferente da nossa pesquisa.
Dentre os alimentos de consumo diário encontramos o arroz, consumido pelo maior número de pessoas, seguido pelo feijão. Representando o grupo das proteínas de origem animal estão o leite e os ovos. Dentre as carnes, o maior consumo semanal foi de frango. O baixo consumo de proteínas pode ser um dos fatores associados ao baixo teor de massa muscular, que aparecem em 31,6\% dos sujeitos estudados na avaliação da circunferência muscular do braço. O consumo de verduras, legumes e frutas é considerado pequeno quando comparado a quantidade destes nutrientes recomendada pelo Guia de Alimentar da População Brasileira ${ }^{8}$.

Os entrevistados dizem que apesar de apreciarem carnes e derivados do leite, não os consomem por serem alimentos demasiadamente fora do orçamento doméstico. Em relação aos legumes, verduras e frutas, admitem que são alimentos de melhor preço. Porém, admitem não utilizá-los por falta de hábito ou pela dificuldade em armazená-los.

Com esses dados, é possível traçar um perfil do indivíduo, da família e da comunidade para a busca de uma prática educativa eficaz, através do incentivo, do autocuidado, do plantio de hortas comunitárias e da utilização de alimentos regionais, gerando, dessa forma, um incentivo a novas políticas públicas que valorizem o bem estar, a qualidade de vida e o bom estado nutricional que podem ser primordiais no controle e erradicação da doença.

Com base nos resultados obtidos passou-se a conhecer melhor a manifestação da doença e seus aspectos sociodemográficos e nutricionais em pacientes dos municípios da Grande Vitória.

O estudo demonstrou que ações simples, como a avaliação de medidas antropométricas, cálculo do IMC e questionários sociodemográfico e alimentar, podem auxiliar na melhoria do acompanhamento a portadores de doenças infecciosas, como é o caso da hanseníase. Estes cuidados devem ser iniciados na atenção primária à saúde com profissionais da equipe de atenção básica devidamente capacitados.

\section{CONFLITO DE INTERESSE}

Os autores declaram não haver nenhum tipo de conflito de interesse no desenvolvimento do estudo.

\section{REFERÊNCIAS}

1. Secretaria de Saúde do Espírito Santo. Gerência Epidemiológica de Vigilância em Saúde do Espírito Santo. Relatório de atividades do Programa Estadual de Controle de Hanseníase. Vitória, ES: Secretaria de Saúde; 2007.

2. Ministério da Saúde. Secretaria de Vigilância em Saúde. Hanseníase no Brasil: dados e indicadores selecionados. Brasília; 2009.

3. Porto CC. Exames Complementares. In: Hungria H, editor. Semiologia médica. Rio de Janeiro: Ed. Guanabara; 2001. p. 977-978.

4. Frisancho AR. New norms of upper limb fat and muscle areas for assessment of nutritional status. Amer J Clin Nutr 1981; 34:2540.

5. Organização Mundial de Saúde. Unidade guia para eliminar a hanseníase como um problema de saúde pública. Genebra: Organização Mundial de Saúde; 1995.

6. Kroke A, Klipstein-Grobusch K, Voss S, Möseneder J, Thielecke F, Noack R, et al. Validation of a self-administered food-frequency questionnaire administered in the European Prospective Investigation into Cancer and Nutrition (EPIC) Study: comparison of energy, protein, and macronutrient intakes estimated with the doubly labeled water, urinary nitrogen, and repeated 24-h dietary recall methods. Am J Clin Nutr 1999; 70:439-447. 
7. Duarte ACG. Avaliação nutricional: aspectos clínicos e laboratoriais. São Paulo: Atheneu; 2007.

8. Ministério da Saúde. Manual clínico de alimentação e nutrição na assistência a adultos infectados pelo HIV. Brasília: Ministério da Saúde; 2006.

9. Garcia ICO. Avaliação nutricional e caracterização sociodemográfica de portadores de hanseníase [dissertação]. [São Paulo]: Universidade de São Paulo; 2006. 138p.

10. Gomes CCD, Pontes MAA, Gonçalves HSG, Penna GO. Perfil clínicoepidemiológico dos pacientes diagnosticados com hanseníase em um centro de referência na região nordeste do Brasil. Investigação Clínica, Epidemiológica, Laboratorial e Terapêutica. An Bras Dermatol 2005; 80(supl 3):S283-288.

11. Rufino Neto A. Tuberculose e o estudo molecular da sua epidemiologia. Rev Cienc Farm Apl 2007; 28:251-257.

12. Madeira ES. Os espaços de transmissão da hanseníase: domicílio, ambiente de trabalho e relações de vizinhanças [dissertação]. [Vitória]: Universidade Federal do Espírito Santo; 2006. 112p.

13. Canicoba M, Feldman N, Lipovetzky S, Moyano O. Evaluación del estado nutricional de pacientes em um hospital con lepra en Argentina. Nutr Hosp 2007; 22:377-381

14. Anselmo MAC. Avaliação do estado nutricional de indivíduos adultos sadios de classe média. Ingestão enérgica e protéica, antropometria, exames bioquímicos do sangue e testes de imunocompetência. Rev Saude Publica 1992; 26:46-53. 